FROM THE ARCHIVES

Editor Alois Pichler

Moira De Iaco

moiradeiaco@gmail.com

\title{
A list of meetings between Wittgenstein and Sraffa
}

\section{Abstract}

This paper presents a list of meetings between Wittgenstein and Sraffa during the period 1929-1951. It was compiled using Sraffa's notes from his pocket diaries and including supplement appointments noted by Wittgenstein. The information - like addresses or names of other speakers - added by Sraffa to some appointments have been here explained in the footnotes. The list shows that the meetings between Wittgenstein and Sraffa can be divided in several different periods.

The following is a list of meetings between Ludwig Wittgenstein and Piero Sraffa during the period 1929-51. The list was compiled using Sraffa's notes of appointments found in his pocket diaries available in the form of digital facsimiles on the Sraffa papers site at Trinity College Library Cambridge. ${ }^{1}$ The list also includes some thirty appointments noted by Wittgenstein in his pocket diaries where they supplement the information contained in Sraffa's diaries. ${ }^{2}$

\footnotetext{
${ }^{1}$ https://www.trin.cam.ac.uk/Piero_Sraffa

${ }^{2}$ I transcribed Sraffa's record of appointments in 2014 while I was a visiting reader at the Wren Library in Cambridge when the digitized diaries were published online. I subsequently compared the data coming from Sraffa's diaries to Lucia Morra's list (Morra 2018) of Wittgenstein's record of appointments with Sraffa, as these were scheduled by Wittgenstein in his pocket diaries, and then added the appointments from Morra's list that were absent in Sraffa's diaries. I would like to thank Alois Pichler for his encouragement and advice
} 
The complete list has a total of 274 appointments. Although some of these scheduled meetings were cancelled by either Sraffa, Wittgenstein, or both, that still gives more than 240 meetings. It would also be natural to assume that additional meetings took place that were not scheduled by either (cf. Morra 2018: 102).

The diaries Sraffa and Wittgenstein used were those generally given by Cambridge University Press to the staff of the University of Cambridge. They are in pocket format and have a black cover. Sraffa's pocket diaries cover the period 1927-81, with the only gap being for the academic year 1932-33. We have 55 of them, with catalogue numbers from E1 to E55. The last three, E53-E55, are travel journals. The first two of these concern travels in Italy, while the last reports a trip to China. The remaining diaries contain short entries. Sraffa jotted down notes, addresses, person names, bibliographic information, and personal information, like "sarto" (tailor), "dentista" (dentist) or references to his state of health, travelling, etc.; these usually appear in Italian. Wittgenstein's diaries extend from 1929 to 1946 and are kept at the Wittgenstein Archive in Cambridge. In general, Wittgenstein noted, in pencil, only his appointments, writing in German or in English. ${ }^{3}$

Wittgenstein's return to Cambridge on 18 January 1929 worried Keynes who carried the initial burden of contact with Wittgenstein, both by promoting his return and taking responsibility for welcoming and helping re-settle him. Keynes wrote thus to his wife on the day of Wittgenstein's arrival in Cambridge:

Well, God has arrived. I met him on the 5.15 train. He has a plan to stay in Cambridge permanently. Meanwhile we have had tea and now I retire to my study to write to you. I see that the fatigue is going to be crushing. But I must not let him talk to me for more than two or three hours a day. (Wittgenstein 2012: 259)

Keynes, already a friend of Sraffa's, may have thought that Wittgenstein - who was always looking for someone to have stimulating conversations with (cf. McGuinness 1986: 86) - needed a partner

regarding this endeavour. The meeting data from Sraffa's diaries were already in 2017 integrated into the Wittgenstein Archives' site where they can be searched and viewed in context with other Wittgenstein metadata. See http:/ /wab.uib.no/sfb/.

${ }^{3} \mathrm{I}$ am in debt to Lucia Morra for this information about Wittgenstein's pocket diaries. 
with enough stamina to engage in sustained discussions and thus provide the stimulation Wittgenstein craved. It is therefore possible that Keynes imagined Sraffa as an interlocutor suitable for Wittgenstein. We do not know to what extent Keynes foresaw that there would be such a long and steadfast friendship between the two, but we must hypothesize that it was thanks to Keynes, and with the complicity of Ramsey who in those years collaborated with Sraffa, that the philosopher and the economist began their acquaintance. They set their first meeting for Sunday 17 February 1929 at $4.30 \mathrm{pm}$ and after one week, on Saturday 23 February, they met again at the same time. As reported by Lucia Morra, despite the fact that Keynes wrote in a letter to his wife that Sraffa and Wittgenstein went canoeing along the river, there are no meetings scheduled between the two again until 27 May 1929 (Morra 2018: 102). During that period, they probably did meet even though this is not recorded in their diaries. These meetings, which have not left a clear trace, may have had a more leisurely and spontaneous nature.

The meetings between Sraffa and Wittgenstein can be divided into several different periods. The first period extends from 1929 to 1933: Wittgenstein and Sraffa met at least 80 times, however their meetings did not become regular until 1931. From 1934 a second period began where the frequency of their meetings slowed down; some communicative difficulties emerged between Wittgenstein and Sraffa as shown in their correspondence (cf. Wittgenstein 2008: 222; McGuinness 2008: 228; De Iaco 2018). In 1938, Wittgenstein and Sraffa's meetings reach a peak of intensity, however once again in 1939 there was a period of decline in the frequency of the meetings. At the end of 1939 Wittgenstein's mood was not at all positive. Political events caused him to sink into depression leaving him unable to carry out his research and thus feeling unproductive and frustrated. At the beginning of 1940 the meetings with Sraffa were affected by Wittgenstein's psychological condition. They met on 3 January 1940, but the appointment of 22 January was cancelled by Wittgenstein who believed they did not have enough to talk about and reach a satisfactory outcome. As a consequence, he later only occasionally asked Sraffa to meet with him. In fact, it turns out that the two then met both on January $23^{\text {rd }}$ and $24^{\text {th }}$. On the $23^{\text {rd }}$ the 
following was recorded in Sraffa's pocket diary E12 at 8:30 a.m.: "Arist. Soc. (Witt. my rooms 8:25)"; they probably went together to the meeting of the Aristotelian Society. On 24 January they met in the afternoon at 4 p.m. In 1943 and 1944 no appointments between Wittgenstein and Sraffa were scheduled. Despite this absence of appointments in their pocket diaries, we can see in a letter from Wittgenstein to Sraffa that the two met at least once, on 19 December 1944 (Wittgenstein 2008: 372). It is evident, however, that in those years communicative difficulties between the two had increased, making it impossible, as Wittgenstein writes in a letter in 1944, to have a fruitful or pleasant conversation (ibid.). In 1946 Sraffa decided to stop meeting Wittgenstein because of the way in which the philosopher wanted to discuss (Monk 1990: 487). After this decision they remained in contact, but from 1947 to 1951 they met less than 10 times.

The list below contains all the appointments Sraffa scheduled to meet with Wittgenstein and, on the basis of Morra 2018, additionally all those appointments which are present in Wittgenstein's diaries ("WPD"), but absent in Sraffa's diaries. The appointments are reported in the language (English or Italian) in which they have been annotated. Commentary footnotes have been added to provide information about place and/or person.

From the annotations of the appointments in the diaries it is possible to deduce important aspects of the meetings. By crossreferencing the appointments with Wittgenstein's and Sraffa's notes or biographical facts the topics of their conversations can in some cases be hypothesized. It is helpful to pay attention to the following aspects of the notes: the progress of the appointments which in some periods are more intense and regular than during others; when the meetings are scheduled - during some periods they are held in the afternoon, during others at lunchtime or in the evening, but rarely in the morning; where they met, when indicated or deducible; the presence of other interlocutors; and, finally, any information useful for understanding the nature of the meeting. 


$\begin{array}{llllll} & \text { Date } & \text { Time } & \text { Place } & \text { With } & \text { Source } \\ 1 & 17.02 .1929 & 16: 30 & \text { LW } & \text { Sraffa E2 } \\ 2 & 23.02 .1929 & 16: 30 & \text { LW } & \text { Sraffa E2 } \\ 3 & 27.05 .1929 & 16: 30 & \text { LW } & \text { Sraffa E2 } \\ 4 & 03.06 .1929 & 16: 30 & \text { LW, Raffaello Piccoli } & \text { Sraffa E2 } \\ 5 & 18.10 .1929 & 16: 30 & \text { LW } & \text { Sraffa E3 } \\ 6 & 02.11 .1929 & 16: 30 & \text { LW (CANCELLED) } & \text { Sraffa E3 } \\ 7 & 04.11 .1929 & 16: 30 & \text { LW } & \text { Sraffa E3 } \\ 8 & 17.11 .1929 & & \text { Heretics } & \\ 9 & 07.03 .1930 & & & \text { LW }(C A N C E L L E D) & \text { Sraffa E3 } \\ 10 & 12.03 .1930 & 16: 30 & & \text { LW, Rossetti }{ }^{6} & \text { Sraffa E3 } \\ 11 & 07.05 .1930 & 16: 30 & & \text { LW } & \text { Sraffa E3 } \\ 12 & 04.06 .1930 & 16: 30 & & \text { LW } & \text { Sraffa E3 } \\ 13 & 14.10 .1930 & 13: 00 & & \text { LW } & \text { Sraffa E3 } \\ 14 & 14.10 .1930 & 13: 00 & \text { LW, Haberler }{ }^{7} & \text { Sraffa E4 } \\ 15 & 24.10 .1930 & 14: 15 & 6 \text { Granch RD }^{8} & \text { LW } & \text { Sraffa E4 }\end{array}$

\footnotetext{
${ }^{4}$ Raffaello Piccoli was Professor of Italian Literature at Cambridge. He wrote a biography of Benedetto Croce. Piccoli, who had already taught in Cambridge from 1913 to 1917, obtained the chair of Italian Literature in February 1929 and moved to England in April. He met Sraffa on 1 May 1929 and on 2 June of the same year, the day before they met in three with Wittgenstein. Alexandra Marjanović hypothesizes that Sraffa and Piccoli had already met in 1927 in Naples or even earlier through their common friend Luigi Einaudi (Marjanović 2005: 58). Sraffa and Piccoli met several times - at least twenty - between 1929 and 1931, especially in 1930. Piccoli died in 1933 and we don't have Sraffa's diary of 1932-1933 - either because it has been lost or Sraffa did not use it for that academic year - so the count of the meetings with Piccoli could be incomplete. The economist and the poet shared, besides nationality and culture, an interest in linguistics and literature. Sraffa introduced Piccoli to Wittgenstein and, after the first meeting, Wittgenstein and Piccoli "met at least thirty times between 11 November 1929 and 12 November 1932" (Marjanović 2005: 58). It is possible that Piccoli approached Wittgenstein with the continental philosophy of Benedetto Croce coming from Hegel. Wittgenstein and Piccoli probably talked about common subjects of interest like language and music.

${ }^{5}$ The Cambridge Heretics Society was founded by C. K. Ogden in 1909. It was a group of Cambridge students and intellectuals. The group continued to meet until 1932. Wittgenstein and Sraffa took part in these meetings. See Monk 1990: 276 ff. for more information on the Heretics Society.

${ }^{6}$ Geoffrey William Rossetti, chairman of The Heretics, belonged to a family of artists and writers.

${ }^{7}$ Haberler was an economist who was a scholar of the Austrian School of Economics. In 1930 he was 30 years old and visiting England to lecture. Sraffa scheduled Haberler's lectures in his diary, so he probably attended them. Considering that Haberler and Wittgenstein were both Austrian, maybe Sraffa thought that they had something in common to discuss and share. It is also possible that Wittgenstein and Haberler had already known one another when both were in Vienna: Haberler was there after his graduation in Economics and Wittgenstein was there for two years to oversee the building of his sister's house which he designed. I am indebted and very grateful to Nerio Naldi for biographical dates about Haberler.

86 Grantchester Road. According to the list of addresses published by the Cambridge University Reporter, in January 1931 Wittgenstein lived at 6 Grantchester Road and it is possible that in October 1930 he already
} 


\section{De laco @ CC-BY}

\begin{tabular}{|c|c|c|c|c|c|}
\hline 16 & 31.10 .1930 & $14: 30$ & 6 Grantch RD, bus 5,31 & LW & Sraffa E4 \\
\hline 17 & 03.11 .1930 & 15:00 & my room & LW & Sraffa E4 \\
\hline 18 & 10.11.1930 & $16: 00$ & & LW & Sraffa E4 \\
\hline 19 & 17.11.1930 & $16: 00$ & & LW & Sraffa E4 \\
\hline 20 & 24.11 .1930 & $16: 30$ & & LW & Sraffa E4 \\
\hline 21 & 01.12 .1930 & $16: 30$ & & $\mathrm{~S}$ & WPD \\
\hline 22 & 05.12 .1930 & $16: 00$ & Union (Read. Room) ${ }^{9}$ & LW & Sraffa E4 \\
\hline 23 & 22.01.1931 & $16: 30$ & & LW & Sraffa E4 \\
\hline 24 & 29.01.1931 & $16: 30$ & & LW & Sraffa E4 \\
\hline 25 & 02.02 .1931 & & & LW rinviare ${ }^{10}$ & Sraffa E4 \\
\hline 26 & 05.02 .1931 & $16: 30$ & & LW (CANCELLED) & Sraffa E4 \\
\hline 27 & 12.02.1931 & $16: 30$ & & $\mathrm{~S}$ & WPD \\
\hline 28 & 20.02 .1931 & $20: 00$ & & LW & Sraffa E4 \\
\hline 29 & 23.02.1931 & $16: 30$ & & LW & Sraffa E4 \\
\hline 30 & 27.02.1931 & $16: 30$ & & LW (CANCELLED) & Sraffa E4 \\
\hline 31 & 02.03 .1931 & $16: 30$ & & LW & Sraffa E4 \\
\hline 32 & 09.03 .1931 & $16: 30$ & & LW (int.) & Sraffa E4 \\
\hline 33 & 27.04 .1931 & $16: 30$ & & LW & Sraffa E4 \\
\hline 34 & 03.05 .1931 & $16: 30$ & & LW & Sraffa E4 \\
\hline \multirow[t]{2}{*}{35} & 10.05 .1931 & $16: 30$ & & $\mathrm{~S}$ & WPD \\
\hline & 17.05.1931 & & & $\mathrm{S}$ & WPD \\
\hline 36 & 30.05 .1931 & $16: 30$ & his room & LW & Sraffa E4 \\
\hline 37 & 31.05 .1931 & & & S (CANCELLED) & WPD \\
\hline 38 & 03.06.1931 & $16: 30$ & & LW & Sraffa E4 \\
\hline 39 & 16.10.1931 & 19:00 & & LW & Sraffa E4/Sraffa E5 \\
\hline 40 & 24.10 .1931 & $17: 00$ & & LW & Sraffa E5 \\
\hline 41 & 31.10 .1931 & $17: 00$ & & LW & Sraffa E5 \\
\hline
\end{tabular}

lived there (Wittgenstein 2003: 53). He had lodged for a year at Mary Alison Hingston's cottage (see Morra 2018: 104).

${ }^{9}$ Reading Room of the Cambridge Union.

10 'postpone' 
Nordic Wittgenstein Review 7 (1) 2018 | pp. 83-99 | DOI 10.15845/nwr.v7i2.3491

\begin{tabular}{|c|c|c|c|c|c|}
\hline 42 & 07.11.1931 & $17: 00 ?$ & & $\mathrm{~S}$ (CANCELLED) & WPD \\
\hline 43 & 11.11.1931 & $17: 00$ & & $\mathrm{LW}$ & Sraffa E5 \\
\hline 44 & 14.11.1931 & $17: 00$ & & $\mathrm{LW}$ & Sraffa E5 \\
\hline 45 & 05.12.1931 & $17: 00$ & $\mathrm{Me}$ & LW & Sraffa E5 \\
\hline 46 & 23.01 .1932 & $16: 30$ & & $\mathrm{LW}$ & Sraffa E5 \\
\hline 47 & 30.01 .1932 & $16: 30$ & $\mathrm{Me}$ & LW & Sraffa E5 \\
\hline 48 & 06.02 .1932 & $16: 30$ & & LW & Sraffa E5 \\
\hline 49 & 13.02 .1932 & $16: 30$ & $\mathrm{Me}$ & $\mathrm{LW}$ & Sraffa E5 \\
\hline 50 & 14.02.1932 & $15: 30$ & & LW & Sraffa E5 \\
\hline 51 & 20.02 .1932 & $16: 30$ & & S & WPD \\
\hline 52 & 27.02.1932 & $16: 30$ & $\mathrm{He}$ & $\mathrm{LW}$ & Sraffa E5 \\
\hline 53 & 08.03.1932 & & & LW & Sraffa E5 \\
\hline 54 & 24.04 .1932 & $16: 30$ & & LW & Sraffa E5 \\
\hline 55 & 01.05.1932 & $16: 30$ & $\mathrm{Me}$ & LW & Sraffa E5 \\
\hline 56 & 08.05 .1932 & $16: 30$ & my room & LW & Sraffa E5 \\
\hline 57 & 15.05 .1932 & $16: 30$ & $\mathrm{He}$ & LW & Sraffa E5 \\
\hline 58 & 22.05 .1932 & $16: 30$ & $\mathrm{Me}$ & $\mathrm{LW}$ & Sraffa E5 \\
\hline 59 & 28.05 .1932 & $16: 30$ & $\mathrm{He}$ & LW & Sraffa E5 \\
\hline 60 & 05.06 .1932 & $16: 30$ & $\mathrm{Me}$ & LW & Sraffa E5 \\
\hline 61 & 10.06 .1932 & & & LW on sat. & Sraffa E5 \\
\hline 62 & 11.06 .1932 & & & S? & WPD \\
\hline 63 & 23.10 .1932 & $16: 30$ & $\mathrm{He}$ & LW & Sraffa E5 \\
\hline 64 & 29.10 .1932 & $16: 30$ & & $S$ & WPD \\
\hline 65 & 06.11 .1932 & $16: 30$ & & LW & Sraffa E5 \\
\hline 66 & 19.11.1932 & $16: 30$ & $\mathrm{Me}$ & LW & Sraffa E5 \\
\hline 67 & 25.11.1932 & & & S & WPD \\
\hline 68 & 04.12 .1932 & & & S & WPD \\
\hline 69 & 29.01 .1933 & $16: 30$ & & S & WPD \\
\hline 70 & 24.02 .1933 & & & S & WPD \\
\hline 71 & 03.03 .1933 & & & S (CANCELLED) & WPD \\
\hline
\end{tabular}




\begin{tabular}{|c|c|c|c|c|c|}
\hline 72 & 11.03 .1933 & & & $\mathrm{~S}$ & WPD \\
\hline 73 & 15.03 .1933 & $15: 30$ & & $\mathrm{~S}$ & WPD \\
\hline 74 & 30.04 .1933 & & & $\mathrm{~S}$ & WPD \\
\hline 75 & 02.05 .1933 & & & $\mathrm{~S}$ & WPD \\
\hline 76 & 07.05 .1933 & & & $\mathrm{~S}$ & WPD \\
\hline 77 & 13.05.1933 & & & S (CANCELLED) & WPD \\
\hline 78 & 20.05 .1933 & & & $\mathrm{~S}$ & WPD \\
\hline 79 & 28.05 .1933 & & & $\mathrm{~S}$ & WPD \\
\hline 80 & 03.06 .1933 & & & $\mathrm{~S}$ & WPD \\
\hline 81 & 25.11 .1933 & $16: 30$ & $\mathrm{Me}$ & LW & Sraffa E6 \\
\hline 82 & 03.12 .1933 & $16: 30$ & $\mathrm{He}$ & LW & Sraffa E6 \\
\hline 83 & 09.12 .1933 & $16: 30$ & $\mathrm{Me}$ & LW & Sraffa E6 \\
\hline 84 & 13.12 .1933 & $16: 30$ & $\mathrm{He}$ & LW & Sraffa E6 \\
\hline 85 & 11.03 .1934 & $16: 30$ & $\mathrm{Me}$ & LW (CANCELLED) ${ }^{11}$ & Sraffa E6 \\
\hline 86 & 16.03 .1934 & $16: 30$ & & LW & Sraffa E6 \\
\hline 87 & 08.05 .1934 & $16: 30$ & & LW (CANCELLED) & Sraffa E6 \\
\hline 88 & 10.05 .1934 & $16: 30$ & & LW & Sraffa E6 \\
\hline 89 & 19.05.1934 & $16: 30$ & $\mathrm{He}$ & LW (CANCELLED) & Sraffa E6 \\
\hline 90 & 26.05 .1934 & $16: 30$ & $\mathrm{He}$ & LW & Sraffa E6 \\
\hline 91 & 31.05 .1934 & & & orologio $48 \mathrm{~L} . \mathrm{W}$. & Sraffa E6 \\
\hline 92 & 15.07.1934 & $16: 30$ & cted 09:00 & LW & Sraffa E6 \\
\hline 93 & 24.01 .1935 & $16: 00$ & & LW & Sraffa E7 \\
\hline 94 & 01.03 .1935 & $16: 30$ & & LW & Sraffa E7 \\
\hline 95 & 03.05 .1935 & $16: 30$ & & LW & Sraffa E7 \\
\hline 96 & 10.05 .1935 & $16: 30$ & his r. & LW & Sraffa E7 \\
\hline 97 & 17.05 .1935 & $16: 30$ & & LW & Sraffa E7 \\
\hline 98 & 24.05 .1935 & $16: 30$ & $\mathrm{He}$ & LW & Sraffa E7 \\
\hline 99 & 06.06 .1935 & & & S (CANCELLED) & WPD \\
\hline
\end{tabular}

\footnotetext{
${ }^{11}$ Sraffa didn't modify this appointment cancelling it in his diary, but we know by a letter of the same day that Wittgenstein decided to postpone their meeting to 16 March (cf. Wittgenstein 2008: 229).
} 


\begin{tabular}{|c|c|c|c|c|c|}
\hline 100 & 07.06 .1935 & $16: 30$ & $\mathrm{Me}$ & $\mathrm{LW}$ & Sraffa E7 \\
\hline 101 & 12.06 .1935 & & & S (CANCELLED) & WPD \\
\hline 102 & 14.06 .1935 & $16: 30$ & $\mathrm{He}$ & LW & Sraffa E7 \\
\hline 103 & 21.06 .1935 & $16: 30$ & $\mathrm{Me}$ & LW & Sraffa E7 \\
\hline 104 & 27.06 .1935 & $17: 00$ & $\mathrm{He}$ & $\mathrm{LW}$ & Sraffa E7 \\
\hline 105 & 28.06 .1935 & $16: 30$ & $\mathrm{He}$ & $\mathrm{LW}$ & Sraffa E7 \\
\hline 106 & 05.07 .1935 & $16: 30$ & $\mathrm{Me}$ & LW & Sraffa E7 \\
\hline 107 & 12.07 .1935 & $16: 30$ & $\mathrm{He}$ & $\mathrm{LW}$ & Sraffa E7 \\
\hline 108 & 20.07 .1935 & $16: 30$ & $\mathrm{Me}$ & LW & Sraffa E7 \\
\hline 109 & 03.08 .1935 & $16: 30$ & & LW (CANCELLED) & Sraffa E7 \\
\hline 110 & 20.10 .1935 & $16: 30$ & & $\mathrm{LW}$ & Sraffa E8 \\
\hline 111 & 25.10 .1935 & 09:00 & $\mathrm{He}$ & $\mathrm{LW}$ & Sraffa E8 \\
\hline 112 & 02.11 .1935 & $16: 30$ & & $\mathrm{~S}$ & WPD \\
\hline 113 & 03.11 .1935 & $16: 30$ & $\mathrm{He}$ & $\mathrm{LW}$ & Sraffa E8 \\
\hline 114 & 10.11.1935 & $16: 30$ & $\mathrm{Me}$ & $\mathrm{LW}$ & Sraffa E8 \\
\hline 115 & 17.11.1935 & $16: 30$ & $\mathrm{He}$ & LW (CANCELLED) & Sraffa E8 \\
\hline 116 & 19.11.1935 & 19:00 & $\mathrm{Me}$ & $\mathrm{LW}$ & Sraffa E8 \\
\hline 117 & 24.11 .1935 & $16: 30$ & $\mathrm{Me}$ & $\mathrm{LW}$ & Sraffa E8 \\
\hline 118 & 03.12 .1935 & 19:00 & $\mathrm{Me}$ & LW & Sraffa E8 \\
\hline 119 & 07.12 .1935 & $18: 15$ & $\mathrm{He}$ & $\mathrm{LW}$ & Sraffa E8 \\
\hline 120 & 11.12.1935 & $16: 30$ & $\mathrm{He}$ & $\mathrm{LW}$ & Sraffa E8 \\
\hline 121 & 20.05 .1936 & $12: 00$ & & $\mathrm{LW}$ & Sraffa E8 \\
\hline 122 & 07.07.1936 & 19.15 & $\mathrm{Me}$ & $\mathrm{LW}$ & Sraffa E8 \\
\hline 123 & 14.07.1936 & 19.15 & $\mathrm{Me}$ & $\mathrm{LW}$ & Sraffa E8 \\
\hline 124 & 15.01.1937 & $11: 00$ & & $\mathrm{LW}$ & Sraffa E9 \\
\hline 125 & 11.06.1937 & $14: 30$ & & $\mathrm{LW}$ & Sraffa E9 \\
\hline 126 & 12.06.1937 & $11: 00$ & & $\mathrm{LW}$ & Sraffa E9 \\
\hline 127 & 14.06.1937 & $16: 30$ & & $\mathrm{LW}$ & Sraffa E9 \\
\hline 128 & 18.07.1937 & & & Sraffa tel 3675 & WPD \\
\hline 129 & 20.06 .1937 & & & Wittg. ? & Sraffa E9 \\
\hline
\end{tabular}




\begin{tabular}{|c|c|c|c|c|}
\hline 130 & 20.07.1937 & 14.30 & $\mathrm{LW}$ & Sraffa E9 \\
\hline 131 & 21.07.1937 & $17: 00$ & LW & Sraffa E9 \\
\hline 132 & 19.01.1938 & $11: 00$ & $\mathrm{LW}$ & Sraffa E10 \\
\hline 133 & 25.01.1938 & $12: 00$ & $\mathrm{LW}$ & Sraffa E10 \\
\hline 134 & 27.01.1938 & 16:00 & LW (medico Vienna) $)^{12}$ & Sraffa E10 \\
\hline 135 & 31.01 .1938 & $12: 00$ & $\mathrm{LW}$ & Sraffa E10 \\
\hline 136 & 04.02 .1938 & $12: 00$ & $\mathrm{LW}$ & Sraffa E10 \\
\hline 137 & 17.03.1938 & 10:00 & S & WPD \\
\hline 138 & 07.05 .1938 & 13:00 & $\mathrm{LW}$ & Sraffa E10 \\
\hline 139 & 10.05 .1938 & $14: 30$ & $\mathrm{LW}$ & Sraffa E10 \\
\hline 140 & 21.05.1938 & 13:00 & LW & Sraffa E10 \\
\hline 141 & 28.05 .1938 & 13:00 & $\mathrm{LW}$ & Sraffa E10 \\
\hline 142 & 03.06 .1938 & 13:00 & $\mathrm{LW}$ & Sraffa E10 \\
\hline 143 & 18.06.1938 & 16:00 & $\mathrm{LW}$ & Sraffa E10 \\
\hline 144 & 22.06 .1938 & $14: 30$ & LW & Sraffa E10 \\
\hline 145 & 27.06.1938 & 18:00 & $\mathrm{LW}$ & Sraffa E10 \\
\hline 146 & 04.07.1938 & $17: 30$ & $\mathrm{LW}$ & Sraffa E10 \\
\hline 147 & 13.07.1938 & $16: 30$ & $\mathrm{LW}$ & Sraffa E10 \\
\hline 148 & 18.07.1938 & $16: 30$ & $\mathrm{LW}$ & Sraffa E10 \\
\hline 149 & 20.07.1938 & 15:00 & LW & Sraffa E10 \\
\hline 150 & 25.07 .1938 & $16: 30$ & LW & Sraffa E10 \\
\hline 151 & 01.08.1938 & $16: 30$ & LW & Sraffa E10 \\
\hline 152 & 08.08.1938 & $13: 15$ & $\mathrm{LW}$ & Sraffa E10 \\
\hline 153 & 15.08.1938 & 13:00 & LW & Sraffa E10 \\
\hline 154 & 17.08.1938 & 13:00 & LW & Sraffa E10 \\
\hline 155 & 22.08 .1938 & 13:00 & $\mathrm{LW}$ & Sraffa E10 \\
\hline 156 & 24.08 .1938 & 13:00 & LW & Sraffa E10 \\
\hline 157 & 07.10 .1938 & 13:15 & LW & Sraffa E10 \\
\hline 158 & 11.10 .1938 & $13: 15$ & $\mathrm{LW}$ & Sraffa E10 \\
\hline
\end{tabular}

12 'doctor Vienna' 
Nordic Wittgenstein Review 7 (1) 2018 | pp. 83-99 | DOI 10.15845/nwr.v7i2.3491

\begin{tabular}{|c|c|c|c|c|}
\hline 159 & 19.10.1938 & $13: 15$ & LW & Sraffa E10 \\
\hline 160 & 24.10 .1938 & $13: 15$ & LW & Sraffa E10/Sraffa E11 \\
\hline 161 & 01.11 .1938 & $14: 15$ & LW & Sraffa E11 \\
\hline 162 & 07.11 .1938 & $13: 15$ & LW (CANCELLED) & Sraffa E11 \\
\hline 163 & 08.11 .1938 & $13: 15$ & LW & Sraffa E11 \\
\hline 164 & 28.11 .1938 & $16: 30$ & LW & Sraffa E11 \\
\hline 165 & 29.11 .1938 & $13: 30$ & LW & Sraffa E11 \\
\hline 166 & 06.12 .1938 & $13: 15$ & $\begin{array}{l}\text { LW (CANCELLED) } \\
\text { overwriting: BANCA }\end{array}$ & Sraffa E11 \\
\hline 167 & 07.12 .1938 & $13: 15$ & LW & Sraffa E11 \\
\hline 168 & 13.12 .1938 & $13: 15$ & LW & Sraffa E11 \\
\hline 169 & 10.01.1939 & $13: 15$ & LW & Sraffa E11 \\
\hline 170 & 17.01.1939 & $13: 15$ & LW & Sraffa E11 \\
\hline 171 & 24.01.1939 & $13: 15$ & LW & Sraffa E11 \\
\hline 172 & 31.01 .1939 & $13: 15$ & LW & Sraffa E11 \\
\hline 173 & 01.02 .1939 & & $\begin{array}{l}\text { LW noted after "Keyn } \\
\text { and together with } \\
\text { "Rosenstein" and "Joa } \\
\text { letter" }\end{array}$ & Sraffa E11 \\
\hline 174 & 07.02.1939 & $13: 15$ & LW & Sraffa E11 \\
\hline 175 & 13.02.1939 & $13: 15$ & LW? & Sraffa E11 \\
\hline 176 & 14.02.1939 & $16: 30$ & LW & Sraffa E11 \\
\hline 177 & 25.02 .1939 & $13: 15$ & LW & Sraffa E11 \\
\hline 178 & 28.02 .1939 & $13: 15$ & LW & Sraffa E11 \\
\hline 179 & 07.03.1939 & $13: 15$ & LW & Sraffa E11 \\
\hline 180 & 31.03 .1939 & $14: 30$ & LW & Sraffa E11 \\
\hline 181 & 03.04.1939 & $14: 30$ & LW & Sraffa E11 \\
\hline 182 & 14.04 .1939 & $13: 00$ & LW & Sraffa E11 \\
\hline 183 & 18.04.1939 & $14: 30$ & LW & Sraffa E11 \\
\hline 184 & 25.04 .1939 & $13: 00$ & LW & Sraffa E11 \\
\hline
\end{tabular}




\begin{tabular}{|c|c|c|c|c|c|}
\hline 185 & 02.05 .1939 & $13: 00$ & & LW & Sraffa E11 \\
\hline 186 & 03.05 .1939 & 09:00 & & $\mathrm{S}$ & WPD \\
\hline 187 & 09.05 .1939 & $13: 00$ & & LW & Sraffa E11 \\
\hline 188 & 16.05 .1939 & $13: 00$ & & LW & Sraffa E11 \\
\hline 189 & 30.05 .1939 & $13: 15$ & & LW & Sraffa E11 \\
\hline 190 & 20.06 .1939 & $13: 15$ & & LW & Sraffa E11 \\
\hline 191 & 10.07.1939 & & & LW & Sraffa E11 \\
\hline 192 & 01.08 .1939 & $12: 00$ & & LW & Sraffa E11 \\
\hline 193 & 02.08 .1939 & $14: 00$ & & LW & Sraffa E11 \\
\hline 194 & 04.09 .1939 & 09:00 & & LW & Sraffa E11 \\
\hline 195 & 20.09 .1939 & $14: 30$ & & LW & Sraffa E11 \\
\hline 196 & 18.10 .1939 & $16: 30$ & & LW & Sraffa E12 \\
\hline 197 & 25.10 .1939 & $16: 30$ & & LW & Sraffa E12 \\
\hline 198 & 01.11 .1939 & $16: 30$ & $\mathrm{He}$ & LW & Sraffa E12 \\
\hline 199 & 08.11 .1939 & $16: 30$ & $17 \mathrm{~B}$ & LW (CANCELLED) & Sraffa E12 \\
\hline 200 & 15.11.1939 & $16: 30$ & 17B & LW & Sraffa E12 \\
\hline 201 & 22.11.1939 & $16: 30$ & $\mathrm{He}$ & LW & Sraffa E12 \\
\hline 202 & 29.11.1939 & $15: 45$ & 17B & LW & Sraffa E12 \\
\hline 203 & 06.12 .1939 & $15: 45$ & & LW & Sraffa E12 \\
\hline 204 & 20.12.1939 & $16: 00$ & $\mathrm{He}$ & LW & Sraffa E12 \\
\hline 205 & 27.12.1939 & $13: 00$ & & LW & Sraffa E12 \\
\hline 206 & 03.01 .1940 & $15: 45$ & & LW & Sraffa E12 \\
\hline 207 & 22.01 .1940 & $20: 25$ & & LW (CANCELLED) & Sraffa E12 \\
\hline 208 & 23.01 .1940 & $20: 30$ & $\begin{array}{l}\text { Arist. Soc. (Witt. my } \\
\text { rooms 8:25) }\end{array}$ & LW & Sraffa E12 \\
\hline 209 & 24.01 .1940 & $16: 00$ & & LW & Sraffa E12 \\
\hline 210 & 07.02 .1940 & $13: 00$ & & LW & Sraffa E12 \\
\hline 211 & 17.02.1940 & $17: 00$ & & LW & Sraffa E12 \\
\hline 212 & 24.02 .1940 & $16: 00$ & $\mathrm{He}$ & LW & Sraffa E12 \\
\hline 213 & 28.02 .1940 & $13: 00$ & $\mathrm{Me}$ & LW & Sraffa E12 \\
\hline
\end{tabular}




\begin{tabular}{|c|c|c|c|c|c|}
\hline 214 & 17.04.1940 & 13:00 & & LW & Sraffa E12 \\
\hline 215 & 24.04 .1940 & $16: 00$ & $\mathrm{He}$ & LW & Sraffa E12 \\
\hline 216 & 06.05 .1940 & & & LW (CANCELLED) & Sraffa E12 \\
\hline 217 & 08.05 .1940 & 13:00 & & $\mathrm{LW}$ & Sraffa E12 \\
\hline 218 & 15.05 .1940 & 13:00 & & LW (CANCELLED) & Sraffa E12 \\
\hline 219 & 29.05 .1940 & $16: 00$ & & LW & Sraffa E12 \\
\hline 220 & 21.10 .1940 & & & LW (CANCELLED) & Sraffa E12 \\
\hline 221 & 22.10 .1940 & 13:00 & & $\mathrm{LW}$ & Sraffa E13 \\
\hline 222 & 30.10 .1940 & 13:00 & & $\mathrm{LW}$ & Sraffa E13 \\
\hline 223 & 13.11.1940 & $18: 30$ & & LW & Sraffa E13 \\
\hline 224 & 20.11 .1940 & $13: 00$ & & LW & Sraffa E13 \\
\hline 225 & 27.11.1940 & & & LW & Sraffa E13 \\
\hline 226 & 24.01.1941 & & & $\mathrm{LW}$ & Sraffa E13 \\
\hline 227 & 04.02 .1941 & $20: 45$ & & $\mathrm{LW}$ & Sraffa E13 \\
\hline 228 & 10.02.1941 & $20: 45$ & & LW & Sraffa E13 \\
\hline 229 & 25.02.1941 & $20: 45$ & & LW & Sraffa E13 \\
\hline 230 & 03.03.1941 & $13: 00$ & & LW & Sraffa E13 \\
\hline 231 & 18.03.1941 & $20: 45$ & & $\mathrm{LW}$ & Sraffa E13 \\
\hline 232 & 20.03 .1941 & $16: 00$ & & $\mathrm{LW}$ & Sraffa E13 \\
\hline 233 & 21.03.1941 & $16: 00$ & & LW (CANCELLED) & Sraffa E13 \\
\hline 234 & 01.04.1941 & $16: 30$ & & LW & Sraffa E13 \\
\hline 235 & 02.04 .1941 & $20: 45$ & & $\mathrm{LW}$ & Sraffa E13 \\
\hline 236 & 04.04 .1941 & $16: 00$ & & $\mathrm{LW}$ & Sraffa E13 \\
\hline 237 & 08.04.1941 & $17: 30$ & & LW & Sraffa E13 \\
\hline 238 & 14.04 .1941 & $20: 45$ & & $\mathrm{LW}$ & Sraffa E13 \\
\hline 239 & 18.04.1941 & $16: 00$ & & LW (or Th? If so, write) & Sraffa E13 \\
\hline 240 & 21.04.1941 & $18: 00$ & & LW & Sraffa E13 \\
\hline 241 & 05.05.1941 & $16: 00$ & da mamma ${ }^{13}$ & $\mathrm{LW}$ & Sraffa E13 \\
\hline 242 & 27.05.1941 & $20: 45$ & & LW (CANCELLED) & Sraffa E13 \\
\hline
\end{tabular}

13 'by mum' 


\begin{tabular}{|c|c|c|c|c|c|}
\hline 243 & 04.06.1941 & $20: 45$ & & LW & Sraffa E13 \\
\hline 244 & 18.06.1941 & $16: 30$ & & LW & Sraffa E13 \\
\hline 245 & 29.07 .1941 & $16: 30$ & my rooms & $\mathrm{LW}$ & Sraffa E13 \\
\hline 246 & 14.08.1941 & $17: 00$ & & LW & Sraffa E13 \\
\hline 247 & 30.09 .1941 & $20: 30$ & & $\mathrm{LW}$ & Sraffa E13 \\
\hline 248 & 10.10.1941 & $20: 45$ & & LW & Sraffa E13 \\
\hline 249 & 11.10 .1941 & $20: 45$ & & $\mathrm{LW}$ & Sraffa E13 \\
\hline 250 & 17.10.1941 & $20: 30$ & & LW & Sraffa E14 \\
\hline 251 & 15.11.1941 & 16:00 & & $\mathrm{LW}$ & Sraffa E14 \\
\hline 252 & 16.11.1941 & & & $\mathrm{LW}$ & Sraffa E14 \\
\hline 253 & 22.11.1941 & & & $\mathrm{LW}$ ? & Sraffa E14 \\
\hline 254 & 29.11.1941 & $16: 00$ & & $\mathrm{LW}$ & Sraffa E14 \\
\hline 255 & 03.04.1942 & $20: 30$ & & $\mathrm{LW}$ & Sraffa E14 \\
\hline 256 & 30.04 .1942 & & 80 Thomas' St ${ }^{14}$ & $\mathrm{LW}$ & Sraffa E14 \\
\hline 257 & 02.06.1942 & $20: 30$ & & $\mathrm{LW}$ & Sraffa E14 \\
\hline 258 & 12.06 .1942 & $21: 00$ & & $\mathrm{LW}$ & Sraffa E14 \\
\hline 259 & 17.06.1942 & $20: 30$ & & $\mathrm{LW}$ & Sraffa E14 \\
\hline 260 & 14.02.1945 & $16: 30$ & & $\mathrm{LW}$ & Sraffa E17 \\
\hline 261 & 08.05.1945 & $17: 00$ & & $\mathrm{LW}$ & Sraffa E17 \\
\hline 262 & 02.06 .1945 & $16: 30$ & & LW & Sraffa E17 \\
\hline 263 & 23.02 .1946 & $18: 00$ & & LW & Sraffa E18 \\
\hline 264 & 02.03 .1946 & $16: 30$ & & $\mathrm{LW}$ & Sraffa E18 \\
\hline 265 & 19.03.1946 & 21:00 & & $\mathrm{LW}$ & Sraffa E18 \\
\hline 266 & 26.03 .1947 & 20: 30 or $21: 00$ & & LW & Sraffa E19 \\
\hline 267 & 09.05.1947 & $21: 00$ & & $\mathrm{LW}$ & Sraffa E19 \\
\hline 268 & 12.11.1947 & 13:00 & & $\mathrm{LW}$ & Sraffa E20 \\
\hline 269 & 13.10 .1948 & $16: 30$ & & $\mathrm{LW}$ & Sraffa E21 \\
\hline 270 & 03.06.1950 & $12: 30-14: 30$ & & LW & Sraffa E22 \\
\hline
\end{tabular}

${ }^{14}$ This road is close to Guy's Hospital where Wittgenstein was working as assistant that year. 
Nordic Wittgenstein Review 7 (1) 2018 | pp. 83-99 | DOI 10.15845/nwr.v7i2.3491

17.06 .1950

Scritto a

Wittgenstein $^{15}$

Sraffa E22

27

27.06.1950

$12: 30-13: 30$

LW

Sraffa E22

272

21.07.1950

13:00

LW

Sraffa E22

273

19.09 .1950

19:00

LW

Sraffa E22

17.06.1950

Scritto a

Wittgenstein

$274 \quad 23.11 .1950 \quad 15: 00$

LW

Sraffa E23

23.02.1951

Wittgenstein in

Sraffa E23

piazza $^{16}$

MORTE DI

29.04.1951

WITTGENSTEIN ${ }^{17}$

Sraffa E23

15 'Written to Wittgenstein'

16 'Wittgenstein in square'; underlined in red. It was probably an important event in Sraffa's opinion, considering Wittgenstein's critical state of health.

17 'Wittgenstein's death'. So written by Sraffa: large and in capital letters. 

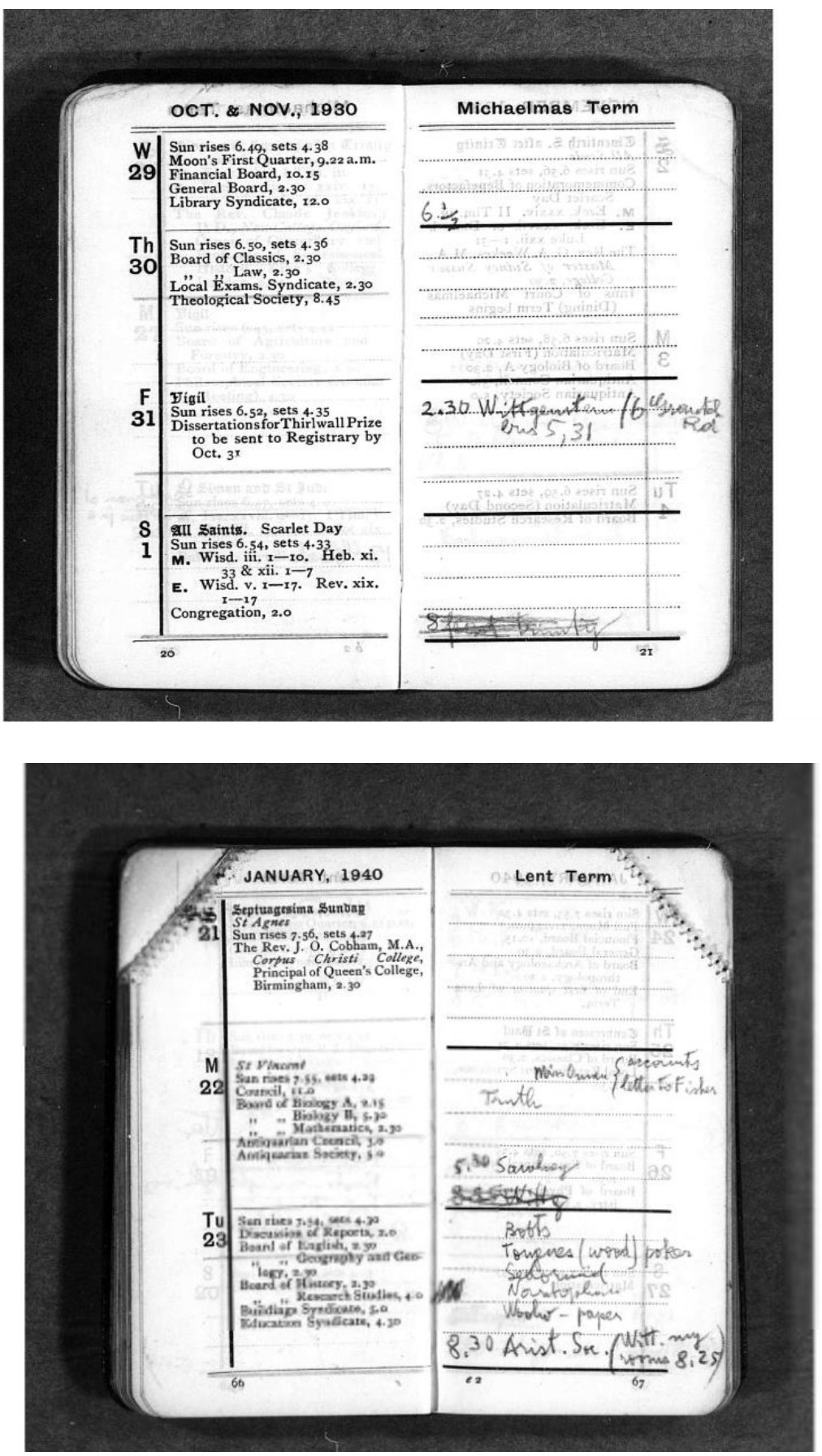

Facsimiles of Sraffa diaries E4 (1930-31) and E12 (1939-40) from the Sraffa Archive at the Wren Library of Trinity College, Cambridge; kindly made available by the Wren Library under the license CC BY-NC-ND. ${ }^{18}$

\footnotetext{
18 http://trin-sites-pub.trin.cam.ac.uk/manuscripts/Sraffa_E4/manuscript.php?fullpage=1\&startingpage=1 and

http://trin-sites-pub.trin.cam.ac.uk/manuscripts/Sraffa_E12/manuscript.php?fullpage=1\&startingpage=1
} 


\section{References}

De Iaco, M., 2018. "Sraffa and Wittgenstein: Steinvorth's testimony, letters and documents". Paradigmi II, forthcoming.

Marjanović, A., 2005. "Introduzione alla vita e alle carte di Raffaello Piccoli: un racconto". Cartevive: Periodico dell'Archivio Prezzolini I, Giugno, pp. 2684.

McGuinness, B., 1986. "Sraffa, Wittgenstein e i problemi del metodo logico". In: R. Bellofiore, ed. Tra teoria economica e grande cultura europea: Piero Sraffa. Milano: Franco Angeli.

McGuinness, B., 2008. "What Wittgenstein owed to Sraffa". In: G. Chiodi and L. Ditta, eds. Sraffa or An Alternative Economics. London: Palgrave Macmillan, pp. 227-235.

Morra, L., 2018. "Friendship and intellectual intercourse between Sraffa and Wittgenstein: a timeline”. In: L. Fiorito, S. Scheall, and C. E. Suprinyak, eds. Research in the History of Economic Thought and Methodology: Including a Symposium on New Directions in Sraffa Scholarship, Vol. 35 B, pp. 101-129.

Venturinha, N., 2012. “Sraffa's Notes on Wittgenstein's «Blue Book»”. Nordic Wittgenstein Review I, pp. 181-191.

Wittgenstein, L., 2003. Public and Private Occasions, eds. J. Klagge and A. Nordmann. Lanham: Rowman \& Littlefield Publishers.

Wittgenstein, L., 2008. Wittgenstein in Cambridge. Letters and Documents 19111951, ed. B. McGuinness. Oxford: Blackwell.

Wittgenstein, L., 2012. Ludwig Wittgenstein: Ein biographisches Album, ed. M. Nedo. München: C.H. Beck.

Monk, R., 1990. Ludwig Wittgenstein: The Duty of Genius. London: Jonathan Cape.

\section{Biographical Note}

Moira De Iaco (b. 1985) received her DPhil in Theory of Language and Science of Signs from the University of Bari. Since 2017 she teaches at the Department of Languages, Literature and Arts at the University of Bari. She is finishing her second $\mathrm{PhD}$ in Philosophy at the Universities of Salento and Cologne working on Sraffa's influence on Wittgenstein. She is the author of several articles and two monographies about Wittgenstein's philosophy (Solipsismo e alterità: Wittgenstein e il mito dell'interiorità and Il segreto dell'arte: Duchamp e Wittgenstein). 